\title{
Mobile Internet Telephony Protocol: An application layer protocol for mobile Internet telephony services
}

\author{
Wanjiun Liao ${ }^{+}$ \\ Department of Electrical Engineering \\ National Taiwan University \\ Taipei, Taiwan \\ Email:wjliao@cc.ee.ntu.edu.tw
}

\begin{abstract}
Internet telephony realizes the transmission of two-way, real-time, synchronous traffic over IP-based networks. Mobile Internet telephony introduces mobility to Internet telephony, allowing one to stay in communications while roaming. In this paper, a novel protocol called Mobile Internet Telephony Protocol (MITP) is proposed. MITP is an application layer protocol based on the client/server model for mobile Internet telephony services. Signaling for mobility management, including registration, call establishment, and roaming, is accomplished through the exchange of defined request and response messages. Much of the message syntax is identical to HTTP/1.1 and SIP/2.0, thereby enabling code reuse and ease of integration of web and SIP servers.
\end{abstract}

Keywords: Mobile Internet Telephony Protocol, Voice over IP, Internet telephony, mobile Internet telephony

\section{Introduction}

Internet telephony, also known as voice over IP, IP telephony, or IPtel, promises to enable real-time two-way voice, and possibly other multimedia data, transmission over the Internet and corporate Intranet. ITU-T Rec. H.323 [1] is the dominant standard for Internet telephony. H.323 specifies technical requirements for voice, data and video communications over packet switched networks without QoS guarantee, and defines the system components and control messages as well as functions for those components. Other efforts toward IP telephony are with the IETF IPtel Working Group (WG). It addresses issues such as the call processing syntax for directing a server in the processing of call signaling, and protocols to locate the appropriate IP telephony gateway for the completion of an IP to POTS, or POTS to IP and then back to POTS call.

In addition to enjoying the potential for lower cost for long-distance calls and for more versatile features, IP telephony may be significantly enhanced by having mobility support, i.e., the ability to maintain communications while moving, another attractive phone service. Consider the following scenario. When a user originates a POTS phone call to an IP telephone on the Internet, the IP host may not be stationary or fixed. It is likely that the IP host is currently on the move from one network to another, or the point of attachment of the IP host has changed. Like cellular phone services, mobile Internet telephone demands seamless roaming while communicating. Under the current version of H.323 and IEFT IPtel WG service scope, however, such mobility capability is still missing.

To realize mobile IPtel services, one may consider to have the underlying network support Mobile IP [2] and run IPtel on top of it. Mobile IP is a network layer protocol that includes some additional control messages for the IP nodes involved, configuring their IP routing tables correctly and reliably for further data delivery to mobile hosts. Voice over IP is a real-time connection-oriented service (RTP, RTCP, TCP, and UDP) over packet-switched IP-based networks. Upon crossing a subnet boundary, a conversation in progress must be handed off; otherwise, the connection would be terminated. Mobile IP, however, is based on connectionless, datagram IP service. No such concept of connection, and therefore handoff, is considered. Besides, a mobility agent defined in Mobile IP is basically a more complicated router with the capability of mobility binding and IP tunneling. With the increasing popularity of personal digital assistants, notebooks, and wireless LAN, there will be additional demand for global connectivity and for mobile IP telephony services, such mobility binding may dramatically increase the burden of these routers. The current trend is toward higher speed IP switching and removing more functionality other than switching out of routers. It is natural to move of mobility binding from the network layer to the upper layer if one carefully examines the mobility binding (or registration in the terminology of Mobile IP) request message defined in Mobile IP, which is encapsulated in an IP/UDP packet.

In this paper, Mobile Internet Telephony Protocol (MITP), an application layer protocol based on the client/server model for mobile Internet telephony service, is proposed. Signaling for mobility management to mobile IPtel, including registration, call establishment, and seamless roaming, is accomplished through the exchanges of request and response messages between user clients and the MITP servers. We will show that through proper call setup and handling signaling with MITP servers, the target address of the called party can be resolved before call

+ This paper is based on work supported by the National Science Council, Taiwan, under grant number NSC 88-2219-E-002-007. 
establishment, enabling the service redirection to be completely handled in the application layer. MITP shares much of message syntax common to HTTP/1.1 [3] and SIP/2.0 [4], thereby allowing code reuse and ease of integration of web and SIP servers. MITP can be operated alone or serve as a complementary protocol for mobile extensions to the IETF Session Initiation Protocol (SIP).

The rest of the paper is organized as follows. The fundamentals of mobile Internet telephony are described after a brief overview of the existing mobility management approaches in Section 2. MITP is proposed in Section 3, including the message definition, signaling messages and operation summaries for mobility management. Finally the concluding remarks and future work are included in Section 4.

\section{Mobile Internet telephony}

Mobile Internet telephony introduces mobility to Internet telephony, allowing one to stay in conversation while moving. Before discussing the requirements of mobile Internet telephony, a brief overview of the existing mobility management approaches is examined first.

\subsection{Mobility management: an overview}

In a digital cellular phone system, say GSM [5], mobility management is realized through Home Location Register (HLR) and Visitor Location Register (VLR) that store the location information of mobile phones. HLR contains location information of users whose primary subscription area is in this service area, while VLR contains location information of users whose primary subscription is with another service area, but presently roaming in this area. A mobile phone initiates a registration when it is first turned on. This operation implicitly informs the corresponding mobility agent (HLR or VLR) of its presence for services. When the mobile phone detects that it in a foreign location area, a location update is performed, in which the local VLR sends the update to the mobile's HLR and acquires relevant information about the visitor from the HLR for further call processing. A mobile phone requires a handoff upon crossing a cell boundary. The connection is handed off from the old cell to the new one. Once the mobile phone has been given a new channel in the new cell, the old channel in the old cell is released and made available to other users in that cell. Location update to an HLR is implicitly performed after a handoff for roaming across service area boundary in a connection-oriented cellular phone system. With such information available, roaming users can be continuously tracked and mobile phone services can be achieved.

With the Mobile IP mechanism, mobility management is performed through the cooperative support of home agent and foreign agent, similar to HLR and VLR in the GSM cellular phone system, respectively. Three major operations provided include agent discovery, registration, and tunneling. Agent discovery is used to advertise the availability of mobility agents for services on each link. It is basically router discovery extended from that defined in ICMP [6]. Registration is designed for location update. When a mobile unit is away from home, a care-of address is temporarily assigned to the visiting mobile unit, either by the foreign agent, or by other means such as DHCP [7]. When a location update is initiated, the care-of address of the mobile unit is sent back to its horne agent for mobility binding ${ }^{1}$. It allows data destined to the mobile unit in the foreign network to be routed correctly via IP tunneling. The home agent intercepts data destined to all registered mobile: units, encapsulates the data into new IP packets with the care-of addresses, and routes to the endpoint as indicated in the care-of addresses.

\subsection{Mobile IPtel fundamentals}

To enable mobile IPtel services, three basic coperations for mobility management must be supported in the system, namely, registration, call establishment, and roaming and hando:.f. The registration process is initiated when one would like to receive mobile IPtel services. It usually follows the agent discovery. Agent discovery is a process used by a mobile IP telephone (Iphone) to ascertain which agent to register with.

The registration process allows a mobile I-phone to inform the affiliated mobility agent of its presence for services and possibly to perform mobility binding if the mobile I-phone is away from home. It is performed before any call cttempt. Services registered may be of type "on-line" or "off-line". It allows a user to be paged for an incoming call, or to use local services through the affiliated mobility agent (such as through the Service Location Protocol [8] to use such local resources as printers). There is no such concept of "on-line" service in Mobile IP. The on-line service is important to mobile IPtel. It is identical to turning a handset on in a digital cellula: phone system and making sure it is ready to receive calls. The information to be registered and saved in a mobility agent includes the mobile I-phone current location address, the port number and transport protocol for receiving calls. If the mobile I-phone is away from its home network, a location update for mobility binding is initiated. Remote redirection, if not authenticated, is widely understood to impose a security problem in the current Internet [9]. It is therefore required that the authentication be verified during a mobility binding process.

Call establishment is the ability to set up a call among participants. Such a call may be a point-to-point two-party call, or a multi-point conference call. The most important cperation for call establishment is callee tracking, since the caller has no idea where the current location of the callee is and if the callee intends, by being registered, to receive a call.

Roaming allows a mobile I-phone to move while communicating. The basic operations for roaming include handoff and possible location update. In the connectionless Mobile IP, no handoff is available because Mobile IP lacks the

\footnotetext{
${ }^{1}$ Mobility binding can be performed by the foreign agent or directly by the mobile unit to its home agent even without an intermedic.ry foreign agent when its care-of address can be obtained dynamically from manners other than from the foreign agent.
} 
concept of connection. [10] has shown that handoffs can be performed through the dynamic join and departure of a multiparty conference. Upon crossing a subnet boundary, a mobile Iphone requires a handoff so that the ongoing conversation is maintained. For a successful handoff, the mobile I-phone registers with the affiliated foreign agent to indicate its presence, followed by a dynamic join and departure of the multi-party conference for handoff. A location update may be performed upon the mobile crossing a service area boundary. The home agent is then informed of the current reachability for mobility binding.

\section{Mobile Internet Telephony Protocol (MITP)}

MITP is an application layer protocol that enables mobile IPtel based on a client-server model. As with other mobility management mechanisms, mobility management in MITP is through the cooperative support of mobility agent servers and user agent clients. Two types of mobility agents are defined: home MITP server and foreign MITP server. The user agent client is the one that resides on a mobile host designed for mobile $\mathrm{IPtel}^{2}$. As in Mobile IP, the home network refers to the network where the mobile I-phone normally resides, and the foreign network, the mobile I-phone may visit. The "network", say home network, is the service area of the corresponding home MITP server, in which the network may be a link, a LAN, or an inter-network with multiple network segments. These MITP servers run on the application layer, waiting and listening to client requests and responding to the requested services.

\subsection{MITP message overview}

Like other client-server model based protocols, MITP messages are exchanged between a client and a server: a request from a client to a server, or a response from a server to a client. Request and response messages are specified with a generic format defined in RFC822 [11] for mobile call handling, with much of the message syntax being identical to HTTP/1.1. It begins with a start-line to indicate a request or a response, followed by one or more header fields to pass related information for processing, an empty line to indicate the end of the headers, and an optional message body, namely,

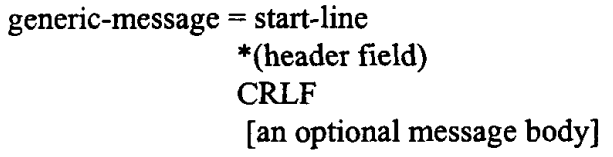

The start-line may either be for a request or for a response, defined, respectively, as follows. Request-line = method SP request-URL SP protocol version CRLF, and Status-line = protocol version SP status code SP reason-phrase CRLF, where SP denotes a space and CRLF denotes the carriage-return linefeed. In the request-line, method denotes the requested service,

\footnotetext{
${ }^{2}$ For simplicity, we will use mobile I-phone to refer to user agent client in a mobile host for mobile IPtel.
}

request-URL indicates the source where the service is provided, and the protocol version describes the MITP version in use. In the status-line (i.e., response-line), the status code indicates the result of the request attempt, and the reason-phrase is the readable description for status code explanation. The requestURL uses MITP addressing with the format of user@host, where the user may be a user name or a telephone number and the host may be a fully-qualified domain name or unique routable IP address, similar to SIP's. The header field, of the form "header-name: value", may carry relevant information to further describe the request/response. It describes the type of information in the header-name followed by a colon, and the information then is specified in the value part. The value part may be in the format of a parameter list separated by a semicolon. The message body may follow the format and definition described in SDP [12]. MITP reuses many headers used in HTTP/1.1 and SIP/2.0. This allows code reuse, and simplifies integration of an MITP server with web and/or SIP servers.

MITP defines four major primitives (methods), including discover, register, call, and bind. The discover method is mainly designed for agent discovery. The mobile I-phone uses register for service registration. The call request is for call establishment, joining or departing an on-going call, and call termination. Basically there are three modes for call handling: create, join, and leave, as indicated in the Call-type header field. "Create" is for a call establishment, "join" means joining an on-going call of the Call-ID, and "leave" means departing from the call. If it is a two-party call, then "leave" means call termination. For call establishment, the connection (voice) between the caller and callee is set up directly by opening a socket with the user IP address, port number and transport protocol indicated by the request. The mobile I-phone uses bind for location update, informing the home MITP server of its reachability for mobility binding.

Due to the space limitation, and for the ease of explanation, only some important header fields are explained, including via, from, to, location, call-ID, expire, call-type. For more complete information, please refer to [13]. The via header records the path taken by the request so for, and can be added by the MITP server during the location tracking and location update processes. As borrowed from [4], the via header "prevents request looping and ensures replies take the same path as the requests. It also assists in firewall traversal and other unusual routing situation." The from and to fields indicate the caller and the callee in a call, respectively, with the format of user@home_host, most likely the email address or URL address for the users. For simplicity, we just show@home_address to indicate home_host. The location field describes where a call is redirected. This often comes with a current location address, port number and transport protocol to announce where and how to establish or to receive the call. The call-ID field shows the call identification. For a multi-party call, all participants should have the same Call-ID. The expire field records the service registration lifetime and binding lifetime for a mobile user. The call-type suggests the appropriate action for request handling. For example, in agent discovery, the call-type of advertisement suggests the requested service be implemented through multicasting or broadcasting, 
the conference through calling $B$ as a new participant (step 3). A request to join multi-party call shares the identical message syntax with call establishment, call termination, and the departure of the call. The join process carries all relevant information about how to make the connection, including the call-ID (19980815@MHb), the current location (at MHa2), port number (3078), and transport protocol (UDP) to be used. $B$ then acks $A$ for join by sending back a response message with a ringing tone, and establishes the connection (step 4). After successfully joining the multi-party call, $A$ (new address) performs location update at its home MITP server for mobility binding (step 5 and 6). This can be performed as in registration. Again, this requires an authentication that is not shown in the figure. $A$ (old address) leaves the conference after $A$ (new address) joins, disconnecting the call with $B$ (step 7), followed by a disconnection reply from $B$ (step 8 ). From the request to depart, $B$ can tell which participant in which call (from the callID) is going to leave. $A$ (old address) then de-registers from MITP server FAa1, thereby releasing any reserved resources (step 9), and de-registers from its home MITP server HAa to remove it from the active binding (step 10). De-registration for both cases share the same message syntax with registration, with the registration lifetime marked "expired". Note that deregistration does not require a reply because the service registration lifetime and binding lifetime expire after some time if no refresh is performed.

\section{Concluding remarks}

In this paper, we have proposed MITP that enables mobile IP telephony services based on the client/service model. The system components are described, the message format is defined, and signaling for mobility management is demonstrated through the exchange of the defined request and response messages. Much of the message syntax is identical or similar to HTTP/1.1 and SIP/2.0, allowing code reuse and ease of integration with web and SIP servers. It moves the mobility support from the network layer to the upper layer, allowing mobile IPtel services to be provided with IP rather than mobile IP. MITP can be operated alone or as a complementary protocol for mobile extensions to the Session Initiation Protocol (SIP). MITP is currently under development at the Multimedia Networking Lab. of the National Taiwan University. The performance and other related issues will be reported in the future.

\section{References}

[1] ITU-T Rec. H.323v2, "Packet Based Multimedia Communications Systems," Jan. 1998.

[2] C. E. Perkins, "IP Mobility Support," RFC 2002, Oct. 1996.

[3] R. Fielding et al. "Hypertext Transfer Protocol - HTTP/1.1," RFC 2068, IETF, Jan. 1997.

[4] M. Handley et al. "SIP: Session Initiation Protocol," IETF, Internet-draft, July 1998 . Work in progress.

[5] S. Redl and M. Weber, "An Introduction to GSM," Artech House, 1995.

[6] S. Deering. "ICMP Router Discovery Messages," RFC 1256,
IETF, Sept. 1991.

[7] R. Droms, "Dynamic Host Configuration Protocol," RFC 2131, Mar. 1997.

[8] J. Veizades et al. "Service Location Protocol," RFC 2165, IETF, June 1997.

[9] C. E. Perkins, "Mobile IP: Design Principles and Practices," Addison-Wesley Wireless Communications Series, 1998.

[10] W. J. Liao, "Mobile Internet Telephony: Mobile Extensions to H.323," IEEE INFOCOM'99, New York.

[11] D. Crocker "Standard for the format of ARPA Internet text messages," RFC 822, IETF, Aug. 1982.

[12] M. Handley and V. Jacobson, "SDP: Session Description Protocol," RFC 2327, IETF, April 1998.

[13] W.J. Liao, "MITP: Mobile Internet Telephony Protocol," Tech. Report, Dept. of EE, National Taiwan University, 1998.

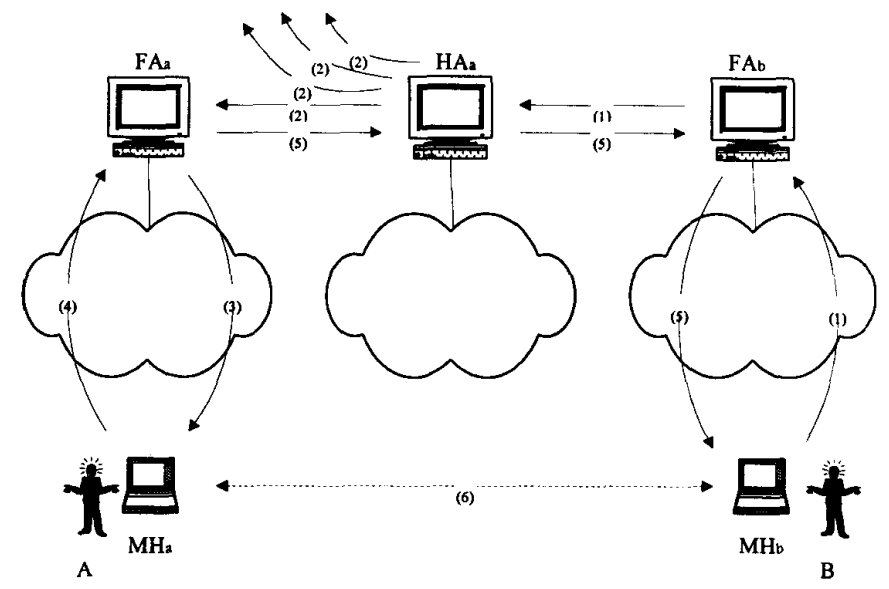

Fig. 3: Call establishment

$\begin{array}{lc}\text { <1>Setup request } & \text { <3>FAa rings A for the incoming call } \\ \text { CALL A@home_address MITP/1.0 } & \text { CALL A@MHa MITP/1.0 } \\ \text { Via: MITP/1.0/UDP FAb } & \text { Via: MITP/1.0/UDP FAa } \\ \text { Via: MITP/1.0/UDP MHb } & \text { Via: MITP/1.0/UDP HAa } \\ \text { From: B@home_address } & \text { Via: MITP/1.0/UDP FAb } \\ \text { To: A@home_address } & \text { Via: MITP/1.0/UDP MHb } \\ \text { Location: B@MHb: 5040;transport=udp } & \text { From: B@home_address } \\ \text { Call-ID: 19980815@MHb } & \text { To: A@home_address } \\ \text { Call-type: Create } & \text { Location: B@MHb: 5040;transport-udp } \\ & \text { Call-ID: 19980815@MHb } \\ \text { <2>Sent paging message for A to all FA's } & \text { Call-type: Create } \\ \text { CALLA@FAa MITP/1.0 } & \\ \text { Via: MITP/1.0/UDP HAa } & \text { <4>A acks the alerting message } \\ \text { Via: MITP/1.0/UDP FAb } & \text { 200 OK MITP/1.0 } \\ \text { Via: MITP/1.0/UDP MHb } & \text { Via: MITP/1.0/UDP FAa } \\ \text { From: B@home_address } & \text { Via: MITP/1.0/UDP HAa } \\ \text { To: A@home_address } & \text { Via: MITP/1.0/UDP FAb } \\ \text { Also: A@FAc,A@FAd } & \text { Via: MITP/1.0/UDP MHb } \\ \text { Location: B@MHb: 5040;transport=udp } & \text { From: B@home_address } \\ \text { Call-ID: } 19980815 @ M H b & \text { To: A@home_address } \\ \text { Call-type: Create } & \text { Call-ID: 19980515@MHb } \\ & \\ & \text { <5 >Ringing to B } \\ & \text { 180 Ringing MITP/1.0 } \\ & \text { Via: MITP/1.0/UDP HAa } \\ & \text { Via: MITP/1.0/UDP FAb } \\ & \text { Via: MITP/1.0/UDP MHb } \\ & \text { From: B@home_address } \\ & \text { To: A@home_address } \\ & \text { Call-ID: 19980815@IHb } \\ & \end{array}$

Fig. 4: Signaling messages for call establishment 